\title{
Arthroscopic Assisted Reduction and Internal Fixation of Tibial Plateau Fractures
}

M.A.Mashhor, H.A.El Begawy, A.M.Halawa and M. K.Mohammed Orthopedic Surgery Dept., Faculty of Medicine, Benha Univ., Benha, Egypt

E-Mail: drmahmoudkhalifa87@gmail.com

\begin{abstract}
Tibial plateau fractures present an important entity in orthopaedic fractures. They may be associated with other knee injuries such as meniscal tears, cruciate ligaments injuries and collateral ligaments damage. The purpose of this study was to reach the latest advances in the different methods of management of tibial plateau fractures using arthroscope and evaluate the results of arthroscopically assisted reduction and internal fixation of tibial plateau fractures on a series of patients clinicaly and radiologicaly. Our study was a prospective case series study that involved 20 patients with tibial plateau fractures presenting to Benha University Hospitals between the period of September 2017 and November 2019.All fractures were evaluated according to the Schatzker classification and there were 2 type I, 7 type II, 7 type III, 2 type IV and 2 type V. According to the clinical Rasmussen score, 15 patients [75\%] had excellent results, [2 type I, 5 types II, 7 types III and 1 type IV], and 5 patients [25\%] had good results [ 2 types II, 1 type IV and 2 types V]. Radiologic results were excellent in 14 cases [70\%] and good in 6 cases [30\%]. There were no patients who had fair or poor results. ARIF of selected tibial plateau fractures allows anatomic reduction and rigid internal fixation with less morbidity than with ORIF, and has the advantage of superior visualization of the entire joint.
\end{abstract}

Keywords: Meniscal, Rasmussen Score, Orthopaedic, Schatzker Classification, Radiologicaly, Pathology.

\section{Introduction}

Tibial plateau fractures affect the proximal tibial metaphysis and articular surface, representing $1.2 \%$ of all fractures and up to $8 \%$ in elderly [1]

They may be related to high-energy trauma especially in young people with good bone density or mild traumatic injury [in elderly individuals with osteoporotic bones]. Approximately 5\% to $10 \%$ of these fractures are related to sports [2].

The role of conservative treatment is limited in the management of fractures of the tibial plateau. Open reduction and internal fixation [ORIF] is the standard treatment option. Several complications may arise from ORIF, such as wound complications and infection [3].

Arthroscopic-assisted reduction and internal fixation [ARIF] is a good alternative to ORIF as it has clear advantages over ORIF. Regarding the fracture reduction, direct and precise visualization of the articular surface is a major advantage because it allows an anatomical reduction without requiring an extensive arthrotomy [4, 5].

Additional intraarticular injuries such as meniscal tears, chondral lesions, or ACL injuries can be diagnosed and treated as usual, with these arthroscopic techniques [6].

Of note, overall morbidity has been reported to be lower with ARIF compared with ORIF due to the decreased invasiveness of the approach. This surgical technique enabled patients to begin early rehabilitation, facilitating return of function and range of motion, and excellent outcome and activity scores. As such, the use of ARIF has been advocated for the management of all tibial plateau fractures $[7,8]$.

\section{Subjects and methods}

Between September 2017 and November 2019, 20 consecutive patients [18 males $\& 2$ females] with closed tibial plateau fractures who had undergone arthroscopic assisted surgery were enrolled in this prospective study.
All of them underwent the same treatment protocol for arthroscopic-assisted surgery with percutaneous screw or buttress plate fixation for closed tibial plateau fractures in Benha university hospitals. All patients were followed up for at least 6 months. The longest follow up was 2 years.

This study involved management of [20] patients with acute tibial plateau fracture.

\subsection{Inclusion criteria}

Acute, Age: more than 16 years old, Patients with fractures [Type I,II,III,IV,V] according to the Schatzker's classification and Closed tibial plateau fracture.

\subsection{Exclusion criteria}

Open fracture dislocation, associated neurovascular injuries, Severely debilitated patients, Highly comminuted plateau and proximal metaphyseal fracture [type V\&VI] and Patients with previous infection of knee joint.

\section{Patient evaluation}

Each patient in this study was carefully assessed clinically in the form of detailed clinical history and through examination.

\subsection{Methods}

All patients were admitted to the Orthopedic and trauma department through the causality or the outpatient clinic and all the patients found in the inclusion criteria were treated with ARIF technique.

\subsection{Primary Management}

Patients were put in a back slab, and the affected limb was elevated until the time of surgery.

\subsection{Time of surgery}

12 patients had been fixed in the first week and 8 
cases there fixation was done in the second week waiting for subsidence of edema.

\subsection{Surgical Techniques Pre-operative preparation}

It was keen to ensure that all needed equipment is available, such as an arthroscopic set, instruments used for conventional knee arthroscopy, Acl C-guide, Kwires, rigid femoral or tibial drills Fig (1).

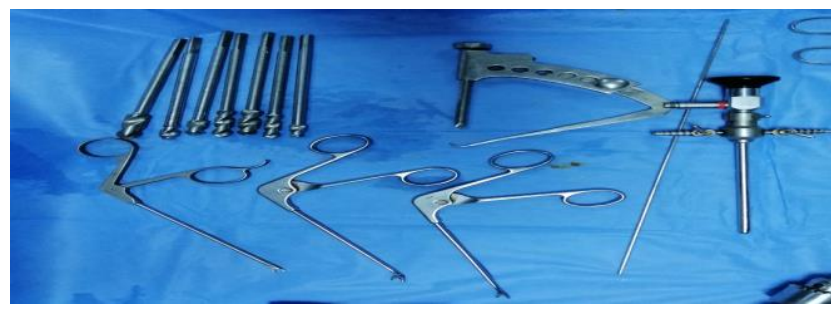

Fig (1) Arthroscopic set and instruments used for ARIF.

\subsection{Patient positioning}

The supine position was used on a flat radiolucent table with a hinged stress post positioned laterally that can be used for medial compartment viewing and can be flipped down when not needed.

\subsection{Operative details}

The standard anterolateral portal was used for

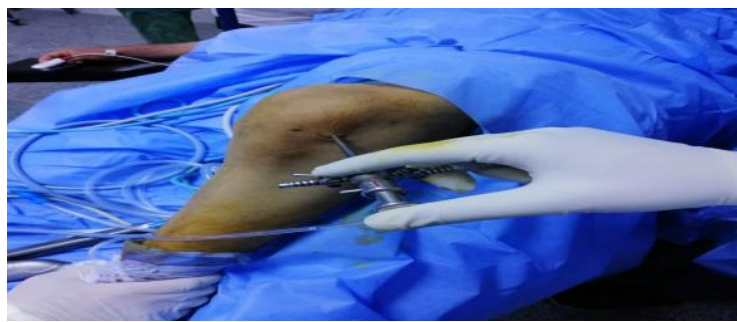

Fig (2) The standard anterolateral portal was used for viewing

\subsection{Post operative management}

The patients received postoperative antibiotic prophylaxis for 4 days. Oral anticoagulation was given and continued until partial weight bearing was allowed. The patients were followed up weekly to check the wound until sutures were removed after 2 weeks. Then, the patients were followed up clinically and radiologically in each monthly visit. The patients were assessed according to Rasmussen's system. Clinical Rasmussen score was used for clinical evaluation of all cases

\section{Rasmussen Score Criteria for Radiological} Assessment [9].

The results are rated as follows:-

- Excellent: 18 points [minimum].

- Good: 12 to 18 points.

- Fair: 6 to 11 points

- Poor: less than 6 points.

\section{Results}

The most frequent types were types II and III [35.0\% for each] followed by I, IV and V [10.0\%] for eachTable (1). viewing Fig (2) Arthroscopic lavage and evacuation of hematoma and loose particles Fig (3). Under arthroscopic guidance, the fractures were reduced and associated intra-articular lesions were addressed appropriately. For split fractures a limited lateral approach, or the joystick technique, was used.Depression fractures were reduced with the aid of an anterior cruciate ligament (ACL) tibial guide.

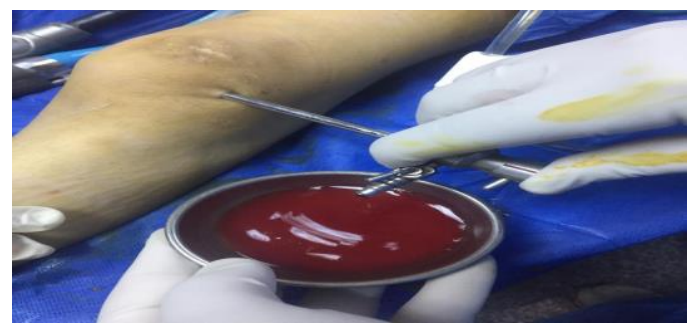

Fig (3) Arthroscopic lavage and evacuation of hematoma and loose particles.

The most frequent type of fixation was cannulated screws in 11 patients [55.0\%] followed by plate and screws in 7 patients [35.0\%]. The least frequent types were double plates \& screws in 1 patient and lateral locked plate in 1 patient [5.0\% for each] Table (2).

Mean time of union was 11 weeks with standard deviation of 2 weeks Table ( 3 ).

As regard radiological Rasmussen score, $70.0 \%$ were excellent, $30.0 \%$ were good and none of the patients had fair or poor scores. Table (4).

As regard clinical Rasmussen score, $75.0 \%$ were excellent, $25.0 \%$ were good and none of the patients had fair or poor scores. Table (5).

There was significant positive correlation between time of union and age $[\mathrm{r}=0.474 \& \mathrm{P}$ value $=0.035]$ Fig (4).

There was significant positive correlation between type of fixation and clinical results $[\mathrm{P}$ value $=0.008]$ Table (6).

Clinical results in all patients with types I and III were excellent but in type $\mathrm{V}$ the results were good Table (7)

There were no complications directly associated 
with arthroscopy. In the 2 patients in which autologous bone grafting was performed, no donor-site morbidity was observed Fig (5).

Table (1) Schatzker type in study population.

\begin{tabular}{lcc}
\hline & & $\mathbf{n}[\%]$ \\
\hline Schatzker type $\mathbf{1 9 8 7}$ & I & $2[10.0]$ \\
& II & $7[35.0]$ \\
III & $7[35.0]$ \\
IV & $2[10.0]$ \\
& V & $2[10.0]$ \\
\hline
\end{tabular}

Table (2)Type of fixation in study population.

\begin{tabular}{lcc}
\hline & & $\mathbf{n}[\%]$ \\
\hline $\begin{array}{l}\text { Type of } \\
\text { Fixation }\end{array}$ & $\begin{array}{c}\text { Cannulated screws } \\
\text { Double plates }\end{array}$ & $11[55.0]$ \\
& \&screws & $1[5.0]$ \\
& Lateral locked plate & $1[5.0]$ \\
& Plate \& screws & $7[35.0]$ \\
\hline
\end{tabular}

Table (3)Time of union in study population.

\begin{tabular}{ll}
\hline & Mean \pm SD \\
\hline Time of union [Weeks] & $11 \pm 2$ \\
\hline
\end{tabular}

Table (4) Radiological Rasmussen score in study population.

\begin{tabular}{lll}
\hline & & $\mathbf{n}[\%]$ \\
\hline Radiological Rasmussen & Excellent & 14 \\
score & & {$[70.0]$} \\
& Good & $6[30.0]$ \\
\hline
\end{tabular}

Table (5) Clinical Rasmussen score in study population.

\begin{tabular}{lll}
\hline & & n [\%] \\
\hline Clinical Rasmussen & Excellent & 15 \\
score & & {$[75.0]$} \\
& Good & $5[25.0]$ \\
\hline
\end{tabular}

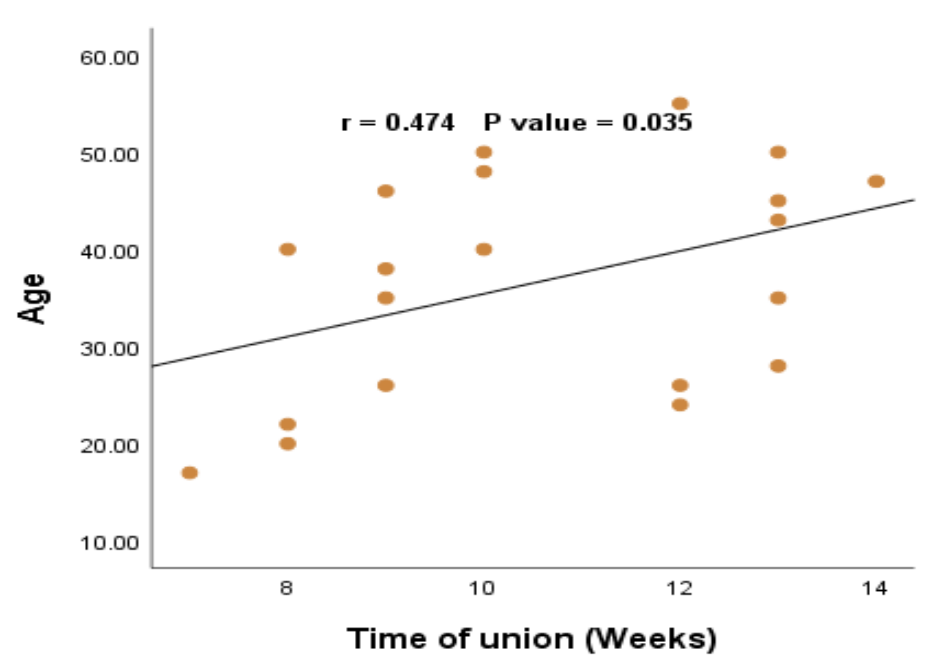

Fig (4) Correlation between time of union and age

Table (6) Effect of type of fixation on Clinical results.

\begin{tabular}{|c|c|c|c|c|}
\hline & & $\begin{array}{l}\text { Cannulated screws } \\
{[\mathrm{n}=11]}\end{array}$ & $\begin{array}{l}\text { Plates } \\
{[\mathrm{n}=9]}\end{array}$ & $\mathrm{P}$ value \\
\hline \multirow[t]{2}{*}{ Outcome } & Excellent & 11 [100.0] & 4 [44.4] & 0.008 \\
\hline & Good & $0[0.0]$ & 5 [55.5] & \\
\hline
\end{tabular}

Table (7) Effect of Schatzker type on clinical results.

\begin{tabular}{|c|c|c|c|c|c|c|}
\hline & & \multicolumn{5}{|c|}{ Schatzker type } \\
\hline \multirow{3}{*}{ Outcome } & & $\mathrm{I}[\mathrm{n}=2]$ & II [n = 7] & III $[n=7]$ & IV $[n=2]$ & $\mathrm{V}[\mathrm{n}=2]$ \\
\hline & Excellent & $2[100.0]$ & $5[71.4]$ & $7[100.0]$ & $1[50.0]$ & $0[0.0]$ \\
\hline & Good & $0[0.0]$ & $2[28.5]$ & $0[0.0]$ & $1[50.0]$ & $2[100.0]$ \\
\hline
\end{tabular}




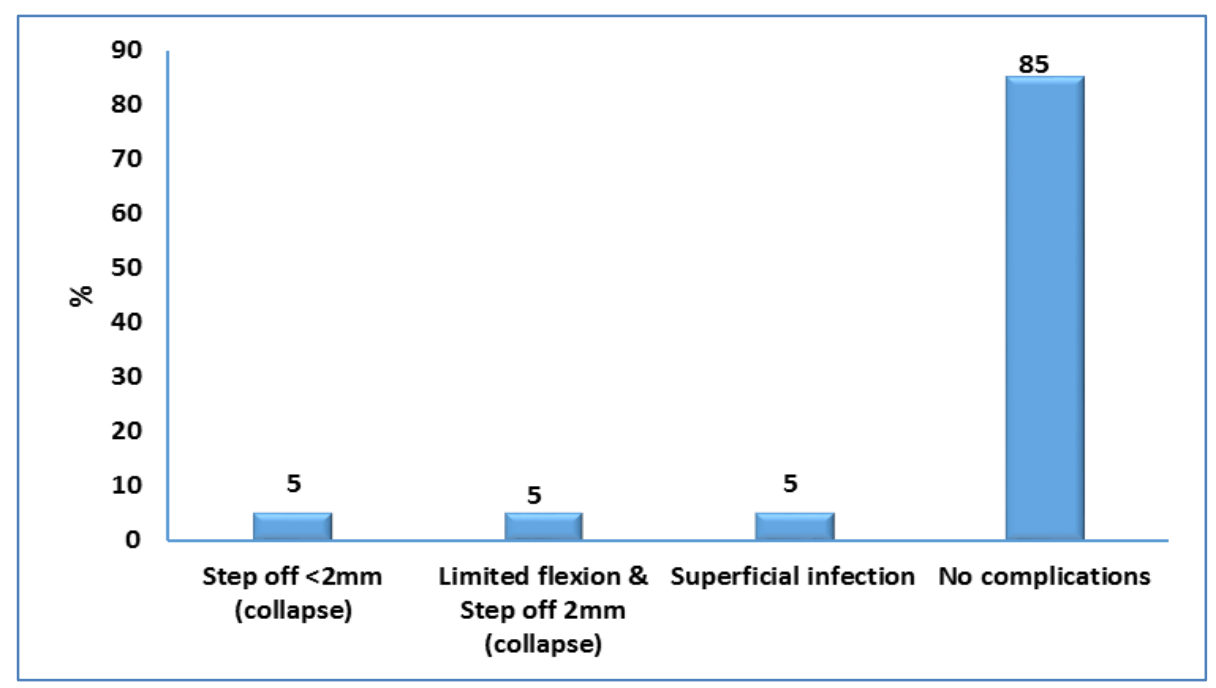

Fig (5) Distribution of complications in study population

\section{Discussion}

Fractures of the tibial plateau represent $1 \%$ of all types of fractures. They should be properly managed. Otherwise, several complications may occur. They may be associated with meniscal tears, cruciate ligaments injuries or collateral ligaments damage [10] .

Dall'Oca $C$ et al [11], compared the results of patients treated with arthroscopic assisted reduction and internal fixation to those of patients treated with the open method. The study was conducted on one hundred patients. They were followed up for seventy-three months on average. There were no differences between the results of both groups in patients with Schatzker type I fractures. However, in type II, III and IV the arthroscopic group showed better results when compared to the open group. In fractures with type $\mathrm{V}$ and VI classification, the results of both groups were poor, but the arthroscopic group had fewer infection rates [11].

Verona M. et al [12] performed a retrospective study comparing the clinical and radiological results of ARIF and ORIF techniques used for Schatzker type I-III fractures in two different groups of patients. The group treated with the arthroscopic technique showed better results in terms of length of hospital stay, clinical scores, and time to full weight-bearing recovery [12].

Although the previous advantages of arthroscopic aided reduction and fixation techniques, not all tibial plateau fractures are amenable to this technique .The arthroscopic treatment of proximal tibial fractures is reserved to those fracture patterns where the direct visualization of the fracture can add a definite value to the fracture reduction process.

In the current study, the arthroscope was first used to assess intra-articular structures, examine for any associated pathology and study the fracture pattern and components. Using the A.C.L guide, a wire was inserted into the fracture site at the center of the depression. A cortical window was created around the wire by the reamer. Then using an impactor, the depressed segment was elevated and carefully reduced. Iliac crest graft was used and impacted under the fracture site in two cases only.

This study showed achievement of union in all cases. The average time to full union was 10.6 weeks. According to the clinical Rasmussen score, 15 patients [75\%] had excellent results, [2 type I, 5 types II, 7 types III and 1 type IV], and 5 patients [25\%] had good results [2 types II, 1 type IV and 2 types V]. Radiologic results were excellent in 14 cases [70\%] and good in 6 cases [30\%]. There were no patients who had fair or poor results.

With reviewing the literature for the applications of arthroscope in treatment of tibial plateau fractures there is a plenty of studies with enough evidence to support the use of arthroscopy to assist in reduction of tibial plateau fractures.

Pogliacomi F. et al [13] treated 18 patients sustaining Schatzker type I, II, III and IV fractures, performing a combined arthroscopic and radioscopic assisted reduction and internal fixation of their tibial plateau fractures. In this retrospective study they evaluated the functional and radiographic results and the value of the combined arthroscopic and radioscopic treatment of tibial plateau fractures. In only three patients they noted fair or poor results. Associated intraarticular injuries were found in 33,3\% of the patients, which were treated after bone fixation. They didn't report any complications related to the arthroscopy procedure [13].

Chiu et al. [14] reported the radiologic and clinical results of posteromedial fractures treated with arthroscopy-assisted reduction and buttress plate and cannulated screw fixation. They operated on twenty-five patients with posteromedial tibial plateau fractures [Schatzker IV, V and VI] with the described technique. The mean follow-up was 86 months [range, 60 to 108 months]. The mean postoperative Rasmussen clinical score was 25.9 [range, 18 to 29]. Among their patients, 11 [44\%] had excellent results, 12 [48\%] had good results [2 type IV, 1 type V, and 9 type VI], and 2 [8\%] had fair results. The mean radiologic score was 15.8 [range, 10 to 18]. The radiologic results were excellent 
or good in $96 \%$ of cases. All 25 fractures achieved successful union, and $92 \%$ had good or excellent clinical and radiologic results. The 3 fracture types did not significantly differ in Rasmussen scores or rates of satisfactory results. Secondary osteoarthritis was noted in 6 injured knees [24\%]. No complications directly associated with arthroscopy were noted in any of the 25 patients [100\%] [14].

\section{Conclusion}

Arthroscopic reduction with rigid internal fixation allows early motion for rehabilitation. Weight bearing is delayed. More complex fracture patterns [Schatzker types V or VI] may not be suitable for arthroscopic treatment. In these cases, ORIF is preferred; the use of arthroscopy in complex proximal tibial fractures has also been suggested, to improve the quality of the reduction.

\section{References}

[1] J. Siegler, B. Galissier, P.S. Marcheix, Percutaneous fixation of tibial plateau fractures under arthroscopy: a medium term perspective. Orthop Traumatol Surg Res , Vol. 97(1), PP.44-50,2011.

[2] R.T. McClellan, C.P. Comstock ,Evaluation and treatment of tibial plateau fractures. Curr Opin Orthop, Vol.10, PP. 10-21,1999.

[3] K. Tilkeridis, G. Kiziridis, S. Tottas, Arthroscopically Assisted Fixation of the Tibial Plateau Fractures. J Bone Res, Vol.6 (188) ,PP.2,2018.

[4] H.J. Lee, H.J. Jung, E.C. Chang, Second-look arthroscopy after surgical treatment of Schatzker type II plateau fractures through the lateral submeniscal approach. Arch Orthop Trauma Surg, Vol.134(4), PP.495-499, 2014.

[5] L. Chen, S.Ma, X. Li , Minimally invasive treatment of tibial plate

[6] au fracture under arthroscopy monitoring.Zhongguo Yi Liao Qi Xie Za Zhi, Vol.38(3), PP.232234,2014 .
[7] E. Ercin, M.G. Bilgili, S.H. Basaran, Arthroscopic treatment of medial femoral condylar coronal fractures and nonunions. Arthrosc Tech, Vol. 2, PP.e413-e415,2013.

[8] G. Burdin , Arthroscopic management of tibial plateau fractures: surgical technique. Orthop Traumatol Surg Res,Vol.99,PP.S208-S218,2012.

[9] J. Kampa, R. Dunlay, R. Sikka, Arthroscopicassisted fixation of tibial plateau fractures: patientreported postoperative activity levels.Orthopedics , Vol.15, PP. 15-30,2016.

[10]P.S. Rasmussen , Tibial condylar fractures. Impairment of knee joint stability as an indication for surgical treatment. J Bone Joint Surg Am, Vol.55, PP.1331-1350,1973.

[11] M. Hohl , I. Part , Fractures of the proximal tibia and fibula. In: Rockwood C, Green D, Bucholz R, eds. Rockwood and Green's Fractures in Adults, Vol. 50, PP. 1725-1757,1992.

[12] C. Dall'Oca, T. Maluta, F. Lavini, Tibial plateau fractures: compared outcomes between ARIF and ORIF. Strategies in Trauma and Limb Reconstruction, Vol. 7(3), PP.163-75,2012.

[13] M. Verona, G . Marongiu , G. Cardoni, Arthroscopically assisted reduction and internal fixation versus open reduction and internal fixation for lateral tibial plateau fractures:a comparative retrospective study Arthroscopy: Journal of Orthopaedic Surgery and Research, Vol.14, PP.155, 2019

[14]F. Pogliacomi, M.A . Verdano , Combined arthroscopic and radio- scopic management of tibial plateau fractures: report of 18 clinical cases. Acta Biomed Ateneo Parmense, Vol.76, PP.107$114,2005$.

[15] C.H. Chiu, C.Y. Cheng, M.C. Tsai, ArthroscopyAssisted Reduction of Posteromedial Tibial Plateau Fractures With Buttress Plate and Cannulated Screw Construct. Arthroscopy, Vol.29, PP.1346-54, 2013. 